\title{
ON THE COINCIDENCE OF THE FEEDBACK NASH AND STACKELBERG EQUILIBRIA IN ECONOMIC APPLICATIONS OF DIFFERENTIAL GAMES
}

\author{
Santiago J. Rubio \\ WP-AD 2002-11
}

Correspondence: University of Valencia. Edificio Departamental Oriental, Avda. de los Naranjos s/n, 46022 Valencia, Spain. E-mail address: Santiago.Rubio@uv.es

Editor: Instituto Valenciano de Investigaciones Económicas, S.A.

FirstEdition July 2002.

DepósitoLegal:யV-2753-2002

IVIE working papers offer in advance the results of economic research under way in order to encourage a discussion process before sending them to scientific journals for their final publication.

* This paper has been accepted from presentation at the Second World Congress of Environmental and Resource Economists, Monterey, CA, June 24-27, 2002. It was finished during my visit at the Department of Economics of the University of Southampton. Financial support from the Ministerio de Ciencia y Tecnología (Spanish Ministry of Science and Technology) under grant BEC2000-1432 is gratefully acknowledged.

** University of Valencia. 


\title{
ON THE COINCIDENCE OF THE FEEDBACK NASH AND STACKELBERG EQUILIBRIA IN ECONOMIC APPLICATIONS OF DIFFERENTIAL GAMES
}

\author{
Santiago J. Rubio
}

\begin{abstract}
A B S T R A C T
In this paper the scope of the applicability of the Stackelberg equilibrium concept in differential games is investigated. Firstly, it is showed that for a class of differential games with state-interdependence the stationary feedback Nash equilibrium coincides with the stationary feedback Stackelberg equilibrium independently of the player being the leader of the game. Secondly, sufficient conditions for obtaining the coincidence between the two equilibria are defined. A review of different economic models shows that this coincidence is going to occur for a good number of economic applications of differential games. This result appears because of the continuous-time setting in which differential games are defined. In this setting the first movement advantage of the leader may disappears and then both equilibria coincide.
\end{abstract}

Keywords: differential games, stationary feedback Nash equilibrium, stationary feedback Stackelberg equilibrium, coincidence.

JEL classification: C73; D62; H41; Q20; Q30 


\section{Introduction}

Differential games have been used in economics to study the strategic interdependence among agents in a dynamic framework. ${ }^{1}$ From the numerous economic applications of differential games we would like to mention the ones developed by Başar, Haurie and Ricci (1985), van der Ploeg and de Zeeuw (1990), Gradus (1991) and Rubio and Escriche (2001). In these papers, devoted to different issues, is explicitly recognized that the feedback Nash equilibrium coincides with the feedback Stackelberg equilibrium. This happens in the modified Lancaster game of capitalism where the feedback Nash equilibrium and the feedback Stackelberg equilibrium, with the workers being the leader, are identical, see Başar, Haurie and Ricci (1985, p. 103); in the model of competitive arms accumulation studied by van der Ploeg and de Zeeuw (1990, p. 133) where for the two players the feedback Nash equilibrium coincides with the feedback Stackelberg equilibrium; in Gradus's (1991, p. 173) model of optimal dynamic profit taxation where the feedback Nash equilibrium and the feedback Stackelberg equilibrium, with the government being the leader, are identical, and also in the model of long-term bilateral interaction between a resource-exporting cartel and a coalition of governments of resource-importing countries developed by Rubio and Escriche (2001, p. 304) where the feedback Nash equilibrium coincides with the feedback Stackelberg equilibrium when the resource cartel acts as the leader of the game.

The aim of this paper is to investigate how general is this coincidence, or in other words, to evaluate the scope of the applicability of the Stackelberg equilibrium concept in differential games. With this objective in mind we define a class of differential games with state-interdependence and we show that for this class of differential games both equilibria are identical independently of the player being the leader of the game. The main characteristic of this class of differential games is that the strategic interdependence among agents appears exclusively through the state variable so that in this framework one agents's decisions only affect the payoff of the rest of agents through his/her influence on the dynamics of the state variable. Then given the continuous-time setting in which differential games are defined the first movement advantage disappears and the two equilibria coincide.

In the second part of the paper we review a set of economic applications of differential games that belong to this class of differential games with state-interdependence, and we check that for all of them the feedback Nash

\footnotetext{
${ }^{1}$ See Dockner et al. (2000) for an excellent survey on differential games applications in economics and management science.
} 
equilibrium is identical to the feedback Stackelberg equilibrium. These examples go from differential games with stock externalities as can be the case of the model of international pollution control developed by Dockner and Long (1993) until the dynamic models of duopoly developed by Fershtman and Kamien (1987) or Reynolds (1987). The conclusion of this review is that the class of differential games defined in this paper cover a good number of interesting economic applications of differential games and that, consequently, for all of them the leadership position does not give any advantage to the leader. This conclusion should not be understood as a criticism of the Stackelberg equilibrium concept but as a limitation of the differential games. This limitation, as we have already pointed out, appears as a consequence of the continuous-time setting in which differential games are defined. ${ }^{2}$

Finally, we define sufficient conditions for getting this result. These conditions guarantee that the reaction functions are orthogonal with respect to the control variables so that an agent's optimal policy only depends on the state variable. In this way we extend our previous result to a broader class of differential games economic applications. We also discuss some examples of differential games that do not fit exactly to the class of differential games with state-interdependence and that present this coincidence between both equilibria. The paper is organized as follows, in the next Section the definitions of a stationary feedback Nash equilibrium and of a stationary Stackelberg equilibrium are presented and the coincidence of both equilibria for a class of differential games with state-interdependence is showed. In Section 3, different economic models where the leadership does not play any role are discussed and in Section 4, sufficient conditions for the equivalence between the two equilibria are presented. Section 5 concludes the paper.

\footnotetext{
${ }^{2}$ All the examples reviewed in Section 2 of this paper are linear quadratic differential games although each of them with a different structure. However, our conclusion about the equivalence of both equilibria is more general and applies for any differential game that presents a state-interdependence.
} 


\section{A class of differential games with state- interdependence}

Let us define a differential game with two players, $i=1$, 2 , where $x(t)$ stands for the vector of state variables, $x(t) \in L$, being $L \subseteq R^{n}$, and $u_{i}(t)$ the vector control variables of the player $i, u_{i}(t) \in U_{i}$, being $U_{i} \subseteq R^{m_{i}}{ }^{3}$ Both players are infinitely lived and have strictly concave, twice differentiable utility functions that depend on the state and control variables, $v_{i}\left(x(t), u_{1}(t), u_{2}(t)\right)$. The dynamics of the sate variables is defined by functions: $f_{j}\left(x(t), u_{1}(t), u_{2}(t)\right), j=$ $1, \ldots, n$, which are twice differentiable. Then the differential game between the two players can be represented by the following optimal control problem

$$
\begin{aligned}
\max _{\left\{u_{i}(t)\right\}} J_{i} & =\int_{0}^{\infty} e^{-r t} v_{i}\left(x(t), u_{1}(t), u_{2}(t)\right) d t \square \\
\text { s.t. } \dot{x}_{j}(t) & =f_{j}\left(x(t), u_{1}(t), u_{2}(t)\right), x_{j}(0)=x_{j 0} \geq 0,
\end{aligned}
$$

where $r$ is the players' rate of discount and $i=1,2$ and $j=1, \ldots, n$.

For the information structure, we consider a feedback or closed-loop information structure. ${ }^{4}$ Given a domain of the state space $L$, player $i^{\prime} s$ feedback strategy is thus expressed as a function on a time-state variable space (i.e., $u_{i}=u_{i}(t, x)$ defined on $\left.[0, \infty) \times L\right)$. But in this paper, we focus on a stationary Markov strategy (i.e., $u_{i}=u_{i}(x)$ defined on $L$ ). Formally, we have the following definition of player $i^{\prime} s$ strategy space.

Definition 1 STATIONARY FEEDBACK STRATEGY SPACE. The stationary feedback strategy space for player $i$ is the set $S_{i}=\left\{u_{i}(x): u_{i}(x)\right.$ is a function of $x \in L$ and $\left.u_{i}(x) \in U_{i}\right\}$.

The feedback strategies describe decision rules that prescribe a value for the control variables as a function of the observed value of the state variables. Players in this case do not commit themselves to a particular path at the outset and can respond to different values they observe. It is now straightforward to present the definition of stationary feedback Nash equilibrium.

Definition 2 STATIONARY FEEDBACK NASH EQUILIBRIUM. A stationary feedback Nash equilibrium is a pair of stationary feedback strategies $\left(u_{1}^{*}, u_{2}^{*}\right) \in S_{1} \times S_{2}$ such that for every possible initial condition $\left(t_{0}, x_{0}\right)$ :

$$
J_{i}\left(u_{i}^{*}, u_{k}^{*}\right) \geq J_{i}\left(u_{i}, u_{k}^{*}\right)
$$

\footnotetext{
${ }^{3}$ Usually, economic considerations determine both the domain of the state space and the domain of the strategy space. For the moment, we do not impose an additional constraint on these domains.

${ }^{4}$ In the traditional differential games literature, see Başar and Olsder (1999), feedback strategies are known as well as Markov strategies.
} 
for every $u_{i} \in S_{i,}(i, k=1,2 ; i \neq k)$.

Feedback strategies provide a subgame perfect equilibrium that is dynamically consistent. Subgame perfectness requires that for every subgame, the restriction of a strategy pair $\left(u_{1}^{*}, u_{2}^{*}\right)$ to the subgame remain an equilibrium in that subgame.

In order to compare this equilibrium concept with the stationary feedback Stackelberg equilibrium concept, we proceed to offer a definition of this equilibrium.

Stipulating an asymmetry in the roles of the players, let player 1 be the leader, announcing a particular path for his control variables, $u_{1}^{o}(t) \in U_{i}$ first, to which player 2 reacts optimally by maximizing $J_{2}\left(u_{1}^{o}, u_{2}\right)$ over $u_{2} \in U_{2}$. Let us assume that the reaction set

$$
R_{2}\left(u_{1}^{o}\right)=\left\{u_{2}^{o} \in U_{2}: J_{2}\left(u_{1}^{o}, u_{2}^{o}\right)=\max _{u_{2} \in U_{2}} J_{2}\left(u_{1}^{o}, u_{2}\right)\right\} \square
$$

is a singleton, so that there exists a unique mapping $T_{2}: U_{1} \rightarrow U_{2}$ with the property

$$
J_{2}\left(u_{1}, T_{2}\left(u_{1}\right)\right)=\max _{u_{2} \in U_{2}} J_{2}\left(u_{1}, u_{2}\right), \square \text { for }\left\lceil\text { every } \llbracket u_{1} \in U_{1} \square\right.
$$

Then, it is easy to give a definition of a stationary feedback Nash Stackelberg equilibrium as follows:

Definition 3 STATIONARY FEEDBACK STACKELBERG EQUILIBRIUM. A stationary feedback Stackelberg equilibrium, with player 1 as the leader, is a pair of stationary feedback strategies $\left(u_{1}^{*}, u_{2}^{*}\right) \in S_{1} \times S_{2}$ such that for every possible initial condition $\left(t_{0}, x_{0}\right)$ :

$$
\begin{aligned}
& u_{1}^{*}=\arg \max _{u_{1} \in U_{1}} J_{1}\left(u_{1}, T_{2}\left(u_{1}\right)\right), \\
& u_{2}^{*}=T_{2}\left(u_{1}^{*}\right) .
\end{aligned}
$$

A stationary feedback Stackelberg equilibrium with player 2 as the leader can be defined analogously, by interchanging the roles of the players. Again this equilibrium is subgame perfect and also dynamically consistent.

Next, we explain how the dynamic programming approach can be used to calculate the equilibria we have just defined.

For the stationary feedback Nash equilibrium, the equilibrium strategies $\left(u_{1}^{*}, u_{2}^{*}\right)$ must satisfy the following Hamilton-Jacobi-Bellman equation

$$
r V_{i}(x)=\max _{\left\{u_{i}\right\}}\left\{v_{i}\left(x, u_{1}, u_{2}\right)+\sum_{j=1}^{n} V_{i j}^{\prime}(x) f_{j}\left(x, \vec{u}_{1}, u_{2}\right)\right\}, i=1,2 \square
$$


where $V_{i}(x)$ is the value for player $i$ of the game that starts at $x$. Note that although in the general case $V_{i}$ is also a function of $t$ and not just of the state variable, it can be shown that if time enters the objective function of the optimal control problem only through the discount term then the value functions do not depend on $t$, see Kamien and Schwartz (1991, p. 238).

The maximization of the right-hand side of the above equation yields

$$
v_{i i}^{\prime}\left(x, u_{1}, u_{2}\right)+\sum_{j=1}^{n} V_{i j}^{\prime}(x) f_{j i}^{\prime}\left(x, u_{1,}, u_{2}\right)=0, \quad i=1,2 . \square
$$

This system of equations implicitly defined the reactions functions of the two players, $u_{1}=T_{1}\left(u_{2}: x\right)$ and $u_{2}=T_{2}\left(u_{1}: x\right)$. Let us assume that functions $v_{i}$ and $f_{j}$ present the sufficient properties to find an unique solution to the

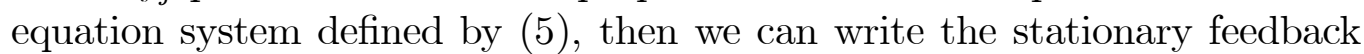
strategies as $u_{1}^{*}=\phi_{1}\left(x, V_{11}^{\prime}(x), \ldots, V_{1 n}^{\prime}(x)\right)$ and $u_{2}^{*}=\phi_{2}\left(x, V_{21}^{\prime}(x), \ldots, V_{2 n}^{\prime}(x)\right)$ or, finally, as functions whose argument is the state variable: $u_{1}^{*}=\chi_{1}(x)$ and $u_{2}^{*}=\chi_{2}(x)$. Substituting them into (4), we obtain

$$
r V_{i}(x)=v_{i}\left(x, \chi_{1}(x), \chi_{2}(x)\right)+\sum_{j=1}^{n} V_{i j}^{\prime}(x) f_{j}\left(x, \chi_{1}(x), \chi_{2}(x)\right), i=1,2
$$

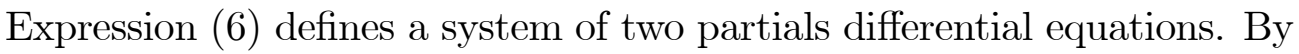
solving this system and finding the value functions $\left(V_{1}(x), V_{2}(x)\right)$, we can also find the equilibrium strategies. Summarizing, we obtain

Proposition 1 A stationary feedback Nash equilibrium is given by a

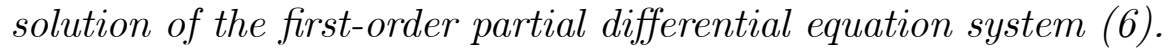

Next, we focus on the stationary feedback Stackelberg equilibrium. Let player 1 be the leader. Then the equilibrium strategy of the leader must satisfy the following Hamilton-Jacobi-Bellman equation

$$
r V_{1}(x)=\max _{\left\{u_{1}\right\}}\left\{v_{1}\left(x, u_{1}, T_{2}\left(u_{1} ; x\right)\right)+\sum_{j=1}^{n} V_{1 j}^{\prime}(x) f_{j}\left(x, u_{1,}, T_{2}\left(u_{1} ; x\right)\right)\right\} . \square
$$

The maximization of the right-hand side of the above equation yields

$$
\begin{gathered}
v_{11}^{\prime}\left(x, u_{1}, T_{2}\left(u_{1} ; x\right)\right)+v_{12}^{\prime}\left(x, u_{1}, T_{2}\left(u_{1} ; x\right)\right) T_{21}^{\prime}\left(u_{1} ; x\right) \\
+\sum_{j=1}^{n} V_{1 j}^{\prime}(x)\left(f_{j 1}^{\prime}\left(x, u_{1}, T_{2}\left(u_{1} ; x\right)\right)+f_{j 2}^{\prime}\left(x, u_{1}, T_{2}\left(u_{1} ; x\right)\right) T_{21}^{\prime}\left(u_{1} ; x\right)\right)=0
\end{gathered}
$$


This equation implicitly defines the strategy of the leader. If we assume that this equation has a unique solution, the explicit strategy of the leader can be written, at the end, as a function of the state variable: $u_{1}^{*}=\psi(x)$. Using this strategy and the reaction function of the follower, the strategy of this player is written as: $u_{2}^{*}=T_{2}\left(u_{1} ; x\right)=T_{2}(\psi(x) ; x)=\omega(x)$. Substituting then into the Hamilton-Jacobi-Bellman equations, we have

$$
r V_{i}(x)=v_{i}(x, \psi(x), \omega(x))+\sum_{j=1}^{n} V_{i j}^{\prime}(x) f_{j}(x, \psi(x), \omega(x)), \quad i=1,2 .
$$

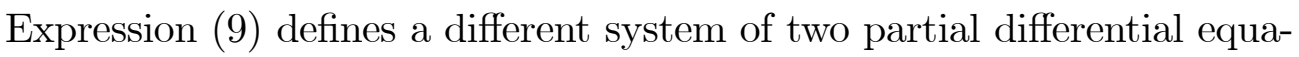
tions. By solving it the value functions are found $\left(V_{1}(x), V_{2}(x)\right)$ which allow to calculate the equilibrium strategies. Summarizing, we present the following result:

Proposition 2 A stationary feedback Stackelberg equilibrium is given

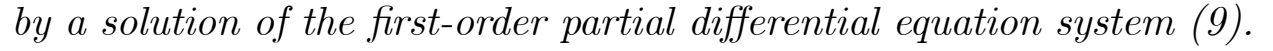

Next, we present a class of differential games with state-interdependence. This class of differential games is characterized by a state equation which depends linearly on the control variables:

$$
f_{j}\left(x, u_{1,}, u_{2}\right)=f_{j}(x)+\alpha_{j \mathfrak{1}} u_{1}+\alpha_{j 2} u_{2,}, j=1, \ldots, n,
$$

where $\alpha_{j 1} \in R^{m_{1}}$ and $\alpha_{j 2} \in R^{m_{2}}$ are two vectors of constants. Moreover, we assume that the objective function of the players does not depend on the other player's control variables:

$$
v_{i}\left(x, u_{1}, u_{2}\right)=v_{i}\left(x, u_{i}\right), \quad i=1,2 . \square
$$

In this class of differential games the strategic interdependence between the players only occurs through the state variable. In other words, the payoffs of a player do not depend directly on the control variable of the other player. The interdependence exists because the decisions of a player affect the pay-offs of the other player, but this effect only appears through the state equation, i.e. through his/her influence on the dynamics of the state variable. In this class of differential games the state variables can be considered as a public good (bad).

For this class of differential games the maximization of the right-hand side of equation $\llbracket(4)$ yields

$$
v_{i i}^{\prime}\left(x, u_{i}\right)+\sum_{j=1}^{n} V_{i j}^{\prime}(x) \alpha_{i j \square}=0, \quad i=1,2 . \square
$$


Now, we have that the two equations are independents with respect to the control variables. This means that the reaction function defined by each equation only depends on the sate variable, or, in other words, that the reaction functions coincide with the stationary feedback strategies.

$$
\begin{aligned}
& u_{1}^{*}=T_{1}(x)=\phi_{1}\left(x, V_{11}^{\prime}(x), \ldots, V_{1 n}^{\prime}(x)\right)=\chi_{1}(x), \\
& u_{2}^{*}=T_{2}(x)=\phi_{2}\left(x, V_{21}^{\prime}(x), \ldots, V_{2 n}^{\prime}(x)\right)=\chi_{2}(x) .
\end{aligned}
$$

As a consequence $T_{21}^{\prime}(x)=T_{12}^{\prime}(x)=0$, and the equation $\square(8)$ is identical to equation (5) and there is no differencebetween the System of partial differential equations $(6)$ and $(9)$ and, consequently, the stationary feedback Stackelberg equilibrium coincides with the stationary feedback Nash equilibrium. Developing this argument, we have that the system of partial differential equations (6) can belwritten now as

$$
r V_{i}(x)=v_{i}\left(x, \chi_{i}(x)\right)+\sum_{j=1}^{n} V_{i j}^{\prime}(x)\left(f_{j}(x)+\alpha_{j 1} \chi_{1}(x)+\alpha_{j 2} \chi_{2}(x)\right), \quad i=1,2 .
$$

For the feedback Stackelberg equilibrium the Hamilton-Jacobi-Bellman equation $\longleftrightarrow(7)$ canbelwritten as

$$
r V_{1}(x)=\max _{\left\{u_{1}\right\}}\left\{v_{1}\left(x, u_{1}\right)+\sum_{j=1}^{n} V_{1 j}^{\prime}(x)\left(f_{j}(x)+\alpha_{j 1} u_{1}+\alpha_{j 2} T_{2}(x)\right)\right\},
$$

which yields

$$
v_{11}^{\prime}\left(x, u_{1}\right)+\sum_{j=1}^{n} V_{1 j}^{\prime}(x) \alpha_{1 j}=0
$$

that is the same equation that we can find in (12) for theplayer 1, and, then, $u_{1}^{*}=\psi(x)=T_{1}(x)$, so that we can conclude that

$$
\begin{aligned}
& u_{1}^{*}=T_{1}(x)=\phi_{1}\left(x, V_{11}^{\prime}(x), \ldots, V_{1 n}^{\prime}(x)\right)=\chi_{1}(x)=\psi(x), \\
& u_{2}^{*}=T_{2}(x)=\phi_{2}\left(x, V_{21}^{\prime}(x), \ldots, V_{2 n}^{\prime}(x)\right)=\chi_{2}(x)=\omega(x),
\end{aligned}
$$

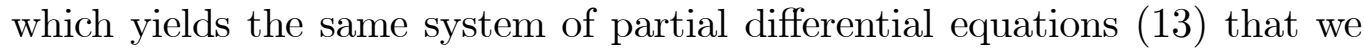
have obtained for the feedback Nash equilibrium. Obviously, this conclusion is independent of which player be the leader. This argument yields the following result:

Proposition 3 For the class of differential games with state externalities defined $\square y \square(10) \square$ and $\square(11)$, $\sqsubset$ the $\square$ stationary $\square$ feedback $\square$ Stackelberg $\sqsubset$ equilibrium $\square$ coincides with the stationary feedback Nash equilibrium independently of the player being the leader of the game. 
This result is a straightforward consequence of the continuous-time setting in which the differential games are defined. In this framework if there does not exist any strategic interdependence through the control variables the first movement advantage disappears and the two equilibria coincide.

As we pointed out in the introduction this kind of equivalence in differential games is already known. However, we do not have a clear idea on the scope of the applicability of the feedback Stackelberg equilibrium in differential games. In the next Section we show that the class of differential games with state-interdependence we have just defined are very common in the economic literature, so that for an important number of economic applications of the differential games theory, the feedback Stackelberg equilibrium is equal to feedback Nash equilibrium. Obviously, this is not a limitation of the Stackelberg equilibrium but the differential games.

\section{Examples}

In this Section we present and discuss different economic models where the leadership does not play any role. ${ }^{5}$ We begin with an example in the field of environmental economics.

\subsection{International pollution control}

A lot of papers have been published in the last years on the international cooperation to control global environmental problems as the greenhouse effect. If we focus on the papers using differential game theory, we can quote the ones published by Brito and Intriligator (1987), Long (1992), van der Ploeg and de Zeeuw (1992) and Dockner and Long (1993). ${ }^{6}$

Brito and Intriligator (1987) and van der Ploeg and de Zeeuw (1992) present a model with $N$ identical players, whereas Long (1992) and Dockner and Long (1993) focus on a model with two identical players. Brito and Intriligator study how the stock (state) externalities can be regulates by Pigouvian taxation of the good that produces the externality in the framework of a feedback Nash equilibrium. van der Ploeg and de Zeeuw (1992) compare the steady-state concentration levels of pollutants in the environment for the efficient equilibrium, the open-loop Nash equilibrium and the

\footnotetext{
${ }^{5}$ A useful source for this Section has been the book published by Dockner et al. (2000).

${ }^{6}$ More recently, Zagonari (1998) and List and Mason (2001) have proposed some extensions of the linear quadratic pollution control model developed by Dockner and Long (1993). Basically they introduce some asymmetry to address different issues. Zagonari studies the effects on pollution of unilateral iniciatives, whereas List and Mason study under what conditions would a decentralized environmental policy be preferable.
} 
feedback Nash equilibrium. They obtain that the feedback strategies increase the inefficiency of the non-cooperative equilibrium in comparison with the open-loop strategies giving a higher steady-state level of pollutant concentration. In Long (1992) an open-loop Stackelberg equilibrium is studied whereas in Dockner and Long (1993) a stationary feedback Nash equilibrium in non-linear strategies is calculated.

Next, we present the model developed by Dockner and Long (1993) and show that the feedback Stackelberg equilibrium coincides with the feedback Nash equilibrium. In Dockner and Long's model there is only one state variable, $x$, that stands for the current stock of pollution, and $u_{1}$ and $u_{2}$, with $\alpha_{11}=\alpha_{12}=1$, represent the pollutant emissions of countries 1 and 2 respectively. Function $f_{1}(x)$ is equal to $-k x$, with $k>0$, that represents the natural purification of the environment. They assume that the net benefit function is quadratic with respect to the pollution stock and emissions. With these simplifications their pollution model becomes a linear quadratic differential game that can be written as follows

$$
\begin{aligned}
& \max _{\left\{u_{i}\right\}} \int_{0}^{\infty} e^{-r t}\left(A u_{i}-\frac{1}{2} u_{i}^{2}-\frac{s}{2} x^{2}\right) d t \\
& \text { s.t. } \dot{x}=u_{1}+u_{2}-k x, \quad x(0)=x_{0} \geq 0 .
\end{aligned}
$$

The equilibrium strategies of the stationary feedback Nash equilibrium of this game must satisfy the following Hamilton-Jacobi-Bellman equation

$r V_{i}(x)=\max _{\left\{u_{i}\right\}}\left\{A u_{i}-\frac{1}{2} u_{i}^{2}-\frac{s}{2} x^{2}+V_{i}^{\prime}(x)\left[E_{i}+E_{j}-k x\right]\right\}, i, j=1,2, i \neq j$,

the f.o.c. for the maximization of the right-hand side of this equation yields

$$
u_{i}^{*}=A+V_{i}^{\prime}(x), \quad i=1,2 \text {. }
$$

Substituting then intol(14), twe obtain

$$
\frac{3}{2} V_{i}^{\prime}(x)^{2}+2 A V_{i}^{\prime}(x)-r V_{i}(x)=\frac{s}{2} x^{2}+k x-\frac{1}{2} A^{2} .
$$

By solving this partial differential equation and finding the value function, Dockner and Long obtain the equilibrium strategies of the stationary feedback Nash equilibrium.

Now, let player 1 be the leader. Then the equilibrium strategy of the leader must satisfy the following Hamilton-Jacobi-Bellman equation

$$
r V_{1}(x)=\max _{\left\{u_{1}\right\}}\left\{A u_{1}-\frac{1}{2} u_{1}^{2}-\frac{s}{2} x^{2}+V_{1}^{\prime}(x)\left[E_{1}+A+V_{2}^{\prime}(x)-k x\right]\right\} .
$$


The maximization of the right-hand side of the above equation yields the same result that the maximization of $\llbracket$ the $r$ right-hand side of equation $(14) \square$ This coincidence bccurs because the reactionfunctions $(15)$ are independent of the control variable of the other player. Obviously, this result does not depend on the symmetry assumption.

\subsection{Common property resources}

In the literature of natural resources one of the first economic applications of dynamic game theory is the paper written by Levhari and Mirman (1980). In this paper, a stationary feedback Nash equilibrium in discrete time is uses to study the interactions between two countries that exploit the same fishery. More recently, Benhabib and Radner (1992) and Dockner and Sorger (1996) have investigated the existence and properties of equilibria for this class of dynamic games proposed by Levhari and Mirman.

In Negri (1989) a differential game approach is applied to the analysis of a common property aquifer. In Negri's groundwater pumping differential game, open-loop and feedback Nash equilibria are compared and it is shown that feedback strategies exacerbate the inefficient private exploitation of the aquifer. Negri's differential game has recently been revisited by Rubio and Casino (2001). In their paper, they adapt the model defined by Gisser and Sánchez (1980) to study the effects of strategic behavior on the efficiency of private groundwater exploitation.

Next, we show that in this model the feedback Stackelberg equilibrium also coincides with the feedback Nash equilibrium. In Rubio and Casino's (2001) differential game $x$ stands for the water table elevation of the aquifer above some arbitrary reference level and $u_{i}$ represents the rate of extraction of the representative farmer with $\alpha_{11}=\alpha_{12}=(\gamma-1)$, being $\gamma, 0<\gamma<1$, the return flow coefficient. They assume that there are $N$ identical farmers pumping water from the same aquifer, however here we will assume that $N=2$. Function $f_{1}(x)$ is equal to escalar $R, R>0$, that stands for the natural recharge of the aquifer. The differential equation which describes the dynamics of the water table is obtained as the difference between natural recharge and net extractions

$$
A S \dot{x}=R+(\gamma-1)\left(u_{1 \square}+u_{2}\right), \square
$$

where $A S$ is area of the aquifer times storativity. Notice that the product $A S \dot{x}$ gives the amount of water pumping from the aquifer in each moment.

The revenues of the farmers are given by a quadratic function of the rate of extraction and the total cost of extraction depends on the rate of extraction 
and the depth of the water table: $C_{i}\left(x, u_{i}\right)=\left(c_{0}+c_{1} x\right) u_{i}$, with $c_{0}>0$ and $c_{1}<0$. Then the farmer maximizes the discounts present value of the stream of profits

$$
\max _{\left\{u_{i}\right\}} \int_{0}^{\infty} e^{-r t}\left[\frac{1}{k} u_{i}^{2}-\frac{g}{k} u_{i}-\left(c_{0}+c_{1} x\right) u_{i}\right] d t
$$

subject to (16) and a given initial water tablelelevation, where $k$ is a negative parameter of the revenue function. For this problem the Hamilton-JacobiBellman equation is

$$
\begin{aligned}
r V_{i}(x)= & \max _{\left\{u_{i}\right\}}\left\{\frac{1}{k} u_{i}^{2}-\frac{g}{k} u_{i}-\left(c_{0}+c_{1} x\right) u_{i}\right. \\
& \left.+V_{i}^{\prime}(x) \frac{1}{A S}\left[R+(\gamma-1)\left(u_{i}+u_{j}\right)\right]\right\}, \square \\
i, j= & 1,2, i \neq j,
\end{aligned}
$$

and the f.o.c. for the maximization of the right-hand side of this equation yields

$$
u_{i}^{*}=\frac{k}{2}\left[\frac{g}{k}+c_{0}+c_{1} x-V_{i}^{\prime}(x) \frac{\gamma-1}{A S}\right], i=1,2 . \square
$$

Substituting, under the symmetry assumption, into (16) the following partial differential equation is obtained

$$
\begin{aligned}
& \frac{3}{4}\left(\frac{\gamma-1}{A S}\right)^{2} V_{i}^{\prime}(x)^{2}-\frac{\gamma-1}{A S} k c_{1} V_{i}^{\prime}(x) x+r V_{i}(x) \\
= & -\frac{k}{4}\left(\frac{g}{k}+c_{0}+c_{1} x\right)^{2}+\frac{1}{A S}\left(R+(\gamma-1)\left(g+k c_{0}\right)\right) .
\end{aligned}
$$

In Rubio and Casino (2001) the equilibrium linear strategies of the stationary feedback Nash equilibrium are calculated by solving this equation.

Stipulating now an asymmetry in the roles of the players, let farmer 1 be the leader. In this case the equilibrium strategy of the leader have to satisfy the following Hamilton-Jacobi-Bellman equation

$$
\begin{aligned}
r V_{1}(x)= & \max _{\left\{u_{1}\right\}}\left\{\frac{1}{k} u_{1}^{2}-\frac{g}{k} u_{1}-\left(c_{0}+c_{1} x\right) u_{1}\right. \\
& \left.+V_{i}^{\prime}(x) \frac{1}{A S}\left(R+(\gamma-1)\left(u_{1}+\frac{k}{2}\left(\frac{g}{k}+c_{0}+c_{1} x-V_{i}^{\prime}(x) \frac{\gamma-1}{A S}\right)\right)\right)\right\} .
\end{aligned}
$$

From the maximization of the right-hand side we get the same strategy that yields the maximization of the $\leftarrow$ right-hand side of ¿equation $(17)$ and ,consequently, we will obtain the same partial differential equation than the one 
that characterizes the stationary feedback Nash equilibrium. This coincidenceloccurs again because the optimal ¿extraction rate given by $(187) \rrbracket$ does not depend on the rate extraction rate of the other farmer.

This result can be easily extended to the case of a renewable resource. Come back to the case studied by Levhari and Mirman. For a restricted common property fishery exploited by two countries, $x$ stands for the fish population and $u_{i}$ represents the harvesting rate of player $i$ with $\gamma=0$. Function $f_{1}(x)$ is now the law of population growth that we can write as $R(x) .{ }^{7}$ Then for the case of a renewable resource the Hamilton-Jacobi-Bellman equation would be written as

$$
\begin{aligned}
r V_{i}(x)= & \max _{\left\{u_{i}\right\}}\left\{\frac{1}{k} u_{i}^{2}-\frac{g}{k} u_{i}-\left(c_{0}+c_{1} x\right) u_{i}\right. \\
& \left.\left.+V_{i}^{\prime}(x)\left[R(x)-u_{i}-u_{j}\right)\right]\right\}, \\
i, j= & 1,2, i \neq j,
\end{aligned}
$$

This equation also gives an optimal policy for the country $i$ that is independent of the harvesting rate of country $j$. Again the two equilibria will be identical. The same occurs if we look at the case of a non-renewable resource as one oilfield exploited by two firms. For this case the above HamiltonJacobi-Bellman equation must be only modified by writing $R(x)=0 .{ }^{8}$

\subsection{Dynamic models of duopoly}

Several papers were published at the end of the eighties proposing a dynamic extension of traditional (static) duopoly theory. This kind of extension was a natural way of explicitly introducing a time-dependent structure into the model in order to analyse the strategic interdependence among firms in a dynamic setting. This literature can be grouped in two blocks.

\subsubsection{Dynamic duopoly with sticky prices}

In 1987, Fershtman and Kamien studied dynamic duopolistic competition under the assumption that the price of a homogeneous product does not

\footnotetext{
${ }^{7}$ An example is the Gomperts' law of population growth: $R(x)=x(a-\rho \ln x)$ used by Dockner, Feichtinger and Mehlmann (1989) in their differential game model of fishery.

${ }^{8}$ Notice that the coincidence between the two equilibria would occur only if the two firms are price-taker. If the firms operate in an oligopolistic market a new interdependence appers through the revenue functions and in that case the reaction fucntion of one firm depends on the other firm's extraction rate resulting in a feedback Stackelberg equilibrium that is different from the feedback Nash equilibrium.
} 
adjust instantaneously to the price indicated by its demand function at the given level of output. In their differential game, price is the state variable and the state equation restricts price to evolve slowly. In 1990 they complete the study of duopolistic competition considering a finite-horizon for the infinitehorizon differential game developed in their previous paper. Later on Tsutsui and Mino (1990), Tsutsi (1996) and Piga (2000) have published different extensions of the Fershtman and Kamien's model. Tsutsui and Mino (1990) compute the stationary feedback Nash equilibrium in non-linear strategies, Tsutsui (1996) introduces capacity constraints and Piga (2000) considers that firms can invest in market-enlarging promotional activities (advertising) which have a public good nature.

In Fershtman and Kamien's (1987) differential game $u_{i}$ represents the $i t h$ firm's output rate and $\alpha_{11}=\alpha_{12}=s$ where $0<s \leq \infty$ denotes the speed in which the price converges to its level on the demand function. Function $f_{1}(x)$ is equal to $s(a-x)$, where $a>0$ stands for the maximum price for the output in the market. Thus, the dynamics of the price is governed by the differential equation

$$
\dot{x}=s\left(a-u_{1}-u_{2}-x\right), \square
$$

where $a-\left(u_{1}+u_{2}\right)$ is the price on the demand function for the given level of output.

The authors assume a quadratic cost function so that the objective function of each firm is

$$
\max _{\left\{u_{i}\right\}} \int_{0}^{\infty} e^{-r t}\left(x u_{i}-c u_{i}-\frac{1}{2} u_{i}^{2}\right) d t
$$

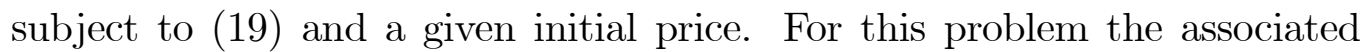
Hamilton-Jacobi-Bellman equation is given by

$$
\begin{gathered}
r V_{i}(x)=\max _{\left\{u_{i}\right\}}\left\{x u_{i}-c u_{i}-\frac{1}{2} u_{i}^{2}+s V_{i}^{\prime}(x)\left(a-u_{i}-u_{j}-x\right)\right\} \\
i=j=1,2, \quad i \neq j,
\end{gathered}
$$

and the f.o.c. for the maximization of the right-hand side of this equation yields

$$
u_{i}^{*}=x-c-s V_{i}^{\prime}(x), i=1,2 .
$$

Substituting these optimal strategies, under the symmetry assumption, into the Hamilton-Jacobi-Bellman equation the following differential equation is obtained.

$$
\frac{3}{2} s^{2} V_{i}^{\prime}(x)^{2}+s(a+2 c-3 x) V_{i}^{\prime}(x)-r V_{i}(x)=-\frac{1}{2}(x-c)^{2} .
$$


By solving this partial differential equation, Fershtman and Kamien find the equilibrium strategies.

In order to calculate the feedback Stackelberg equilibrium of the game we assume that firm 1 acts as the leader, being the Hamilton-Jacobi-Bellman equation

$r V_{1}(x)=\max _{\left\{u_{1}\right\}}\left\{x u_{1}-c u_{1}-\frac{1}{2} u_{1}^{2}+s V_{i}^{\prime}(x)\left(a-u_{1}-\left(x-c-s V_{2}^{\prime}(x)\right)-x\right)\right\}$.

Again, we obtain the same optimal policy than the one obtained for the feedback Nash equilibrium and, consequently, the two equilibria coincide.

\subsubsection{Dynamic duopoly with adjustment costs}

In other papers the dynamics appear in the model from production or investment adjustment costs. See Fershtman and Muller (1984), Reynolds (1987) and (1991), Maskin and Tirole (1987), Driskill and McCafferty (1989) and Dockner (1992). In Fershtman and Muller (1984) the existence and stability of the open-loop Nash equilibrium are addressed. In Reynolds' (1987) paper it is assumed that the reduced form net revenue (sales revenue less production costs) is a function of the capacities of the two firms and that there exist non-linear costs to adjust the capacity. In Reynolds' (1991) paper, he extends his analysis of the duopoly to the case of an oligopoly with $N$ firms. On the other hand, in Driskill and McCafferty (1989) and Dockner (1992), the intrinsic dynamics of duopolistic competition arises from production adjustment costs. In these papers firms incur costs associated with how fast they change their level of output so that the levels of output become the state variables and the rates of change of output act as the control variables. ${ }^{9}$

In this Section we limit ourselves to comment the differential game defined by Reynolds (1987). In his model there are two state variables which represent the capacity of the two firms, so that $u_{i}$ stands for the ith firm's gross investment with $\alpha_{11}=\alpha_{22}=1$ and $\alpha_{12}=\alpha_{21}=0$. Functions $f_{j}(x)$ are equal to $-\delta x_{i}$ where $\delta \geq 0$ is the exponential rate of depreciation, then the state equations are given by

$$
\dot{x}_{i \sqsubseteq u_{i}}-\delta x_{i,} \quad i=1,2 . \square
$$

The author assumes a quadratic function for the adjustment costs of the capacity, and a linear demand function and technology that allows him to

\footnotetext{
${ }^{9}$ In Maskin and Tirole (1987) a discrete time Cournot model with adjustment costs and alternating moves is presented. The authors assume that firms cannot change their output plans for a given finite time (they are able to commit themselves for finite periods) and move alternatingly.
} 
write the net revenue function as a function of capacities. The result is that the objective for firm $i$ is

$$
\max _{\left\{u_{i}\right\}} \int_{0}^{\infty} e^{-r t}\left(x_{i}\left(a-x_{i}-x_{j}\right)-q u_{i}-\frac{c}{2} u_{i}^{2}\right) d t, i=j=1,2, i \neq j,
$$

subject to the state equations (20) anda given/initial[levelffor capacities. [For this dynamic optimization problem the associated Hamilton-Jacobi-Bellman equation is written as follows

$$
\begin{aligned}
r V_{i}\left(x_{i}, x_{j}\right)= & \max _{\left\{u_{i}\right\}}\left\{x_{i}\left(a-x_{i}-x_{j}\right)-q u_{i}-\frac{c}{2} u_{i}^{2}\right. \\
& \left.+V_{i i}^{\prime}\left(x_{i}, x_{j}\right)\left(u_{i}-\delta x_{i}\right)+V_{i j}^{\prime}\left(x_{i}, x_{j}\right)\left(u_{j}-\delta x_{j}\right)\right\}, \\
i= & j=1,2, \quad i \neq j,
\end{aligned}
$$

and the f.o.c. for the maximization of the right-hand side of this equation yields

$$
u_{i}^{*}=\frac{1}{c}\left(-q+V_{i i}^{\prime}\left(x_{i}, x_{j}\right)\right), \quad i=j=1,2, \quad i \neq j .
$$

The following system of partial differential equations emerges after substituting for $u_{i}^{*}$ and $u_{j}^{*} \operatorname{in}\lfloor(21)$

$$
\begin{array}{r}
\frac{V_{i i}^{\prime}\left(x_{i}, x_{j}\right)^{2}}{2 c}-\left(\frac{q}{c}+\delta x_{i}\right) V_{i i}^{\prime}\left(x_{i}, x_{j}\right)+\frac{1}{c} V_{j j}^{\prime}\left(x_{i}, x_{j}\right) V_{i j}^{\prime}\left(x_{i}, x_{j}\right) \\
-\left(\frac{q}{c}+\delta x_{j}\right) V_{i j}^{\prime}\left(x_{i}, x_{j}\right)-r V_{i}\left(x_{i}, x_{j}\right)=-\left(x_{i}\left(a-x_{i}-x_{j}\right)+\frac{q^{2}}{2 c}\right) .
\end{array}
$$

The solution of this equation, under the assumption of symmetry, allows to Reynolds to obtain the equilibrium strategies.

If firm 1 is the leader of the game the corresponding Hamilton-JacobiBellman equation is

$$
\begin{aligned}
r V_{1}\left(x_{1}, x_{2}\right)= & \max _{\left\{u_{1}\right\}}\left\{x_{1}\left(a-x_{1}-x_{2}\right)-q u_{1}-\frac{c}{2} u_{1}^{2}+V_{11}^{\prime}\left(x_{1}, x_{2}\right)\left(u_{1}-\delta x_{1}\right)\right. \\
& \left.+V_{12}^{\prime}\left(x_{1}, x_{2}\right)\left(\frac{1}{c}\left(-q+V_{22}^{\prime}\left(x_{1}, x_{2}\right)\right)-\delta x_{2}\right)\right\}, \square
\end{aligned}
$$

and we obtain that

$$
u_{1}^{*}=\frac{1}{c}\left(-q+V_{11}^{\prime}\left(x_{1}, x_{2}\right)\right) .
$$

Then by substituting iit into $(23) \longleftarrow$ we get a partial differential equation iiden-

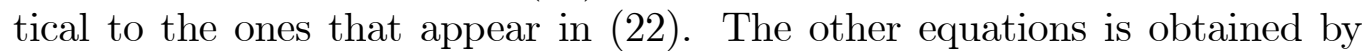
substitution of the optimal policy function of the leader into the HamiltonJacobi-Bellman equation of the follower. 


\subsection{Dynamic voluntary provision of public goods}

In this Section we comment Fershtman and Nitzan's (1991) paper that can be viewed as an extension of the results obtained in the dynamic duopoly literature, we have just presented, to the theory of voluntary provision of public goods. ${ }^{10}$

In Fershtman and Nitzan's differential game among $N$ identical agents that jointly carry out a certain project by making continuous contributions of some input, $x$ stands for the stock of total contributions and $u_{i}$ is interpreted as the contribution rate of agent $i$ being the parameters $\alpha_{1}=\ldots=\alpha_{N}=1$. In this presentation we simplify the model assuming that $N=2$. Function $f(x)$ is equal to $-\delta x$ where $\delta \geq 0$ is the exponential rate of depreciation. Then the total contribution $x$ accumulates over time according to

$$
x_{i \sqsubset}=u_{1 \square}+u_{2}-\delta x, \square
$$

The cost of contribution and the benefit from the public good for agent $i$ are given by quadratic functions. They further assume that the objective function of each agent is to maximize its discounted utility, i.e.

$$
\max _{\left\{u_{i}\right\}} \int_{0}^{\infty} e^{-r t}\left(\alpha\left(a x-b x^{2}\right)-\frac{c}{2} u_{i}^{2}\right) d t, \square i \mp 1,2, \square
$$

subject to $(24)$ for a given initial value of the total $\sqsubset$ contributions. $\alpha$ in $\llbracket(25)$ represents how subtractable is the good. For $\alpha=1 / 2$ the project involves the production of a typical private good. If $\alpha=1$, the good can be interpreted as a pure public good. Using the value function approach the equilibrium strategies must satisfy the following Hamilton-Jacobi-Bellman condition

$$
r V_{i}(x)=\max _{\left\{u_{i}\right\}}\left\{\alpha\left(a x-b x^{2}\right)-\frac{c}{2} u_{i}^{2}+V_{i}^{\prime}(x)\left(u_{i}+u_{j}-\delta x\right)\right\}, i=1,2, i \neq j .
$$

The maximization of the right-hand side yields $u_{i}^{*}=V_{i}^{\prime}(x) / c$ and by substitution of this optimal policy, assuming that the two value functions are identical, the following partial differential equation is obtained

$$
\frac{3}{2 c} V_{i}^{\prime}(x)^{2}-\delta x V_{i}^{\prime}(x)-r V_{i}(x)=-\alpha\left(a x-b x^{2}\right) .
$$

By solving this equation and finding the value function $V_{i}(x)$, Fershtman and Nitzan find the equilibrium strategies.

\footnotetext{
${ }^{10}$ More recently, Itaya and Shimomura (2001) have used a similar differential game to study the sign of the conjectural variations at the steady state in the dynamic private provision of public goods.
} 
Now, let us assume that player 1 enjoys a first-movement advantage. Then the equilibrium strategy of the leader must satisfy

$$
r V_{1}(x)=\max _{\left\{u_{1}\right\}}\left\{\alpha\left(a x-b x^{2}\right)-\frac{c}{2} u_{1}^{2}+V_{1}^{\prime}(x)\left(u_{1}+\frac{1}{c} V_{2}^{\prime}(x)-\delta x\right)\right\},
$$

and we get that $u_{1}^{*}=V_{1}^{\prime}(x) / c$, exactly the same optimal policy function than the one obtained for the feedback Nash equilibrium.

\subsection{Competitive arms race}

The conflict between West and East over arms accumulation has been studied using differential game theory by Brito (1972), Simaan and Cruz (1975), Deger and Sen (1984) and, more recently, by van der Ploeg and de Zeeuw (1990). In these models the welfare of one country depends on the level of security which is perceived to be an increasing function of its own weapon stock and a decreasing function of the foreign weapon stock. In Brito (1972) and Deger and Sen (1984) the open-loop Nash equilibrium of the game is characterized and in Simaan and Cruz (1975) and van der Ploeg and de Zeeuw (1990), the feedback Nash equilibrium.

In van der Ploeg and de Zeeuw's differential game there are two state variables which represent the weapon stocks of the two regions. $u_{i}$ denotes the level of governments investment in arms with $\alpha_{11}=\alpha_{22}=1$ and $\alpha_{12}=$ $\alpha_{21}=0$. Functions $f_{j}(x)$ are equal to $-\delta x_{i}$ where $\delta \geq 0$ is the depreciation rate. The arms accumulation evolves according to

$$
x_{i \sqsubset u_{i}}-\delta x_{i,} i=1,2 . \square
$$

The authors assume that utility is separable in defence and that defence depends on the difference between the weapon stocks of the two regions. For a quadratic approximation of the utility function, each region maximizes

$$
\begin{aligned}
& \max _{\left\{u_{i}\right\}} \int_{0}^{\infty} e^{-r t}\left(\theta_{0}+\theta_{1} u_{i}-\frac{1}{2} \theta_{2} u_{i}^{2}+\theta_{3}\left(x_{i}-x_{j}\right)-\frac{1}{2} \theta_{4}\left(x_{i}-x_{j}\right)^{2}\right) d t, \\
& i=1,2, i \neq j,
\end{aligned}
$$

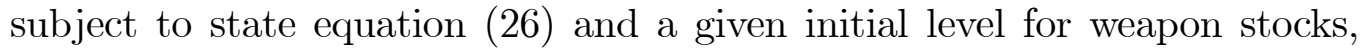
being all parameters $\theta$ positive except $\theta_{1}$ that must be negative. For this dynamic optimization problem the associated Hamilton-Jacobi-Bellman equation is written as follows

$$
r V_{i}\left(x_{i}, x_{j}\right)=\max _{\left\{u_{i}\right\}}\left\{\theta_{0}+\theta_{1} u_{i}-\frac{1}{2} \theta_{2} u_{i}^{2}+\theta_{3}\left(x_{i}-x_{j}\right)\right.
$$




$$
\begin{aligned}
& -\frac{1}{2} \theta_{4}\left(x_{i}-x_{j}\right)^{2}+V_{i i}^{\prime}\left(x_{i}, x_{j}\right)\left(u_{i}-\delta x_{i}\right) \\
& \left.+V_{i j}^{\prime}\left(x_{i}, x_{j}\right)\left(u_{j}-\delta x_{j}\right)\right\}, \square
\end{aligned}
$$

where $i=j=1,2, \quad i \neq j$.

The maximization of the right hand side of this equation yields

$$
u_{i}^{*}=\frac{1}{\theta_{2}}\left(\theta_{1}+V_{i i}^{\prime}\left(x_{i}, x_{j}\right)\right), i=j=1,2, i \neq j .
$$

The following system of partial differential equations appears when substituting for $u_{i}^{*}$ and $u_{j}^{*}$ in $(27)$

$$
\begin{aligned}
& \frac{2 \theta_{2}-\theta_{3}}{2 \theta_{2}^{2}} V_{i i}^{\prime}\left(x_{i}, x_{j}\right)^{2}+\left(\frac{\theta_{1}\left(2 \theta_{2}-\theta_{3}\right)}{\theta_{2}^{2}}-\delta x_{i}\right) V_{i i}^{\prime}\left(x_{i}, x_{j}\right) \\
+ & \frac{1}{\theta_{2}} V_{i j}^{\prime}\left(x_{i}, x_{j}\right) V_{j j}^{\prime}\left(x_{i}, x_{j}\right)+\left(\frac{\theta_{1}}{\theta_{2}}-\delta x_{j}\right) V_{i j}^{\prime}\left(x_{i}, x_{j}\right)-r V_{i}\left(x_{i}, x_{j}\right) \\
= & -\theta_{0}-\frac{\theta_{1}^{2}\left(2 \theta_{2}-\theta_{3}\right)}{2 \theta_{2}^{2}}-\theta_{3}\left(x_{i}-x_{j}\right)+\frac{1}{2} \theta_{4}\left(x_{i}-x_{j}\right)^{2} .
\end{aligned}
$$

By solving this system, under the assumption that both value functions are identical, van der Ploeg and de Zeeuw find the equilibrium strategies.

If now we assume that the West, player number 1, could be the leader of this game, its equilibrium strategy should be satisfy

$$
\begin{aligned}
r V_{1}\left(x_{1}, x_{2}\right)= & \max _{\left\{u_{1}\right\}}\left\{\theta_{0}+\theta_{1} u_{1}-\frac{1}{2} \theta_{2} u_{1}^{2}+\theta_{3}\left(x_{1}-x_{2}\right)\right. \\
& -\frac{1}{2} \theta_{4}\left(x_{1}-x_{2}\right)^{2}+V_{11}^{\prime}\left(x_{1}, x_{2}\right)\left(u_{1}-\delta x_{1}\right) \\
& \left.+V_{12}^{\prime}\left(x_{1}, x_{2}\right)\left(\frac{1}{\theta_{2}}\left(\theta_{1}+V_{22}^{\prime}\left(x_{1}, x_{2}\right)\right)-\delta x_{2}\right)\right\},
\end{aligned}
$$

but from this equation the same equilibrium strategy than in the feedback Nash equilibrium is obtained.

To close this Section we would like to highlight that the coincidence between the two equilibria concepts in this class of differential games does not depend on the asymmetry assumption. This assumption is just used in order to reduce the system of partial differential equations to one and facilitate in this way the calculation of the equilibrium strategies. In other words, if any asymmetric version of the differential games presented in this Section could be solved the coincidence between the two equilibria would occur as well. 


\section{Control externalities}

In this Section we show that the coincidence between the two equilibria studied in this paper can also appear in games where the strategic interdependence also comes through the control variables. In fact, it is easy to find sufficient conditions for getting this result in differential games. Let us assume that

Remark 1 (Assumption) The first derivatives $\partial v_{i} / \partial u_{i}$ and $\partial f_{j} / \partial u_{i}, j=$ $1, \ldots, n$ are independents of $u_{k}, i=k=1,2$ and $i \neq k$.

Then we have that

Proposition 4 If Remark 1 is satisfied, the feedback Stackelberg equilibrium coincides with the feedback Nash equilibrium independently of which player acts as the leader.

Proof. For the general problem (DG), the equilibrium Nash strategies must Satisfy (4). If $\llbracket$ Assumption 1 holds, the maximization of the right-hand side 0 f $(4)$ yields

$$
v_{i i}^{\prime}\left(x, u_{i}\right)+\sum_{j=1}^{n} V_{i j}^{\prime}(x) f_{j i}^{\prime}\left(x, u_{i}\right)=0, \quad i=1,2,
$$

and we find that $u_{1}^{*}=T_{1}(x)$ and $u_{2}^{*}=T_{2}(x)$. Now, we can follow the proof of Prop. 3 to conclude that the feedback Stackelberg equilibrium is identical to the feedback Nash equilibrium.

A corollary from this result is

Corollary 1 Let us assume that $\partial v_{i} / \partial u_{i}$ and $\partial f_{j} / \partial u_{i}, j=1, \ldots, n$ are independents of $u_{k}$ but that the contrary is not true for $i=k=1,2$ and $i \neq k$, then there exists only one feedback Stackelberg equilibrium for the game where player $i$ is the leader and player $k$ is the follower.

Proof. If the first derivatives $\partial v_{i} / \partial u_{i}$ and $\partial f_{j} / \partial u_{i}$ are independent of $u_{k}$ we know that $u_{i}^{*}=T_{i}(x)$. If the contrary is not true, the maximization of the right-hand side of the Hamilton-Jacobi-Bellman equation of player $k$ yields

$$
v_{k k}^{\prime}\left(x, u_{i}, u_{k}\right)+\sum_{j=1}^{n} V_{k j}^{\prime}(x) f_{j k}^{\prime}\left(x, u_{i}, u_{k}\right)=0, \quad i=k=1,2, i \neq k,
$$


which implicitly defines the reaction function of player $k, u_{k}^{*}=T_{k}\left(u_{i} ; x\right)$. Given these relationships betwwen the players, player i can influence the behavior of player $k$, but this is not the case for player $k$. Consequently, in the unique feedback Stackelberg equilibrium that the game admits player $k$ is the follower and player $i$ the leader.

In order to illustrate these results we now comment two examples of asymmetric differential games.

\subsection{Optimal dynamic profit taxation}

In Gradus (1991) the optimal profit taxation for a welfare maximizing government in a dynamic framework is studied using the differential game theory. In his model, $x$ stands for the capital stock of the representative firm. The capital accumulation evolves according to

$$
x=u_{2}-\delta x,
$$

where $u_{2}$ is the investment of the firm and $\delta \geq 0$ the capital depreciation rate. For our notation player 2 is the representative firm. The net revenues depend on the corporate tax rate and on the capital stock according to the following expression: $\left(1-u_{1}\right) a x$, where $u_{1}$ denotes the corporate rate and $a$ is a constant that represents the marginal productivity of capital. In the paper it is assumed that the production function presents constant returns to scale. The objective of the firm is to maximize its discounted stream of net cash flows

$$
\max _{\left\{u_{2}\right\}} \int_{0}^{\infty} e^{-r t}\left(\left(1-u_{1}\right) a x-u_{2}-\varphi\left(u_{2}\right)\right) d t
$$

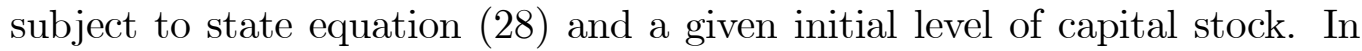
this expression, $\varphi\left(u_{2}\right)$ represents the internal adjustment costs of the firm.

On the other hand, it is assumed that the government, player 1, has the same utility function as the representative consumer, that public consumption will be financed from profit taxation, and that there is no debt. The utility depends on private and public consumption and assuming a CobbDouglas utility function the objective of the government can be written as follows

$$
\max _{\left\{u_{1}\right\}} \int_{0}^{\infty} e^{-\beta t}\left(\alpha \ln \left(\left(1-u_{1}\right) a x+w h x-u_{2}-\varphi\left(u_{2}\right)\right)+(1-\alpha) \ln u_{1} a x\right) d t
$$

subject to State equation(28) and the initial level of capital stock. In/this expression $w$ stands for the real wage rate and $h x$ gives the number of employed 
workers. Notice that in this differential game the government's discount rate is different of the firm's interest rate.

Now, if we check whether the Axiom 1 holds for this differential games we find that

$$
\frac{\partial v_{1}}{\partial u_{1}}=-\frac{\alpha a x}{\left(1-u_{1}\right) a x+w h x-u_{2}-\varphi\left(u_{2}\right)}+\frac{1-\alpha}{u_{1}}, \frac{\partial f}{\partial u_{1}}=0,
$$

and

$$
\frac{\partial v_{2}}{\partial u_{2}}=-1-\varphi^{\prime}\left(u_{2}\right), \quad \frac{\partial f}{\partial u_{2}}=1
$$

So that there exists a unique feedback Stackelberg equilibrium which is different from the feedback Nash equilibrium. For this equilibrium player 2, the firm, is the leader and player 1, the government, is the follower. Notice that $\partial v_{1} / \partial u_{1}$ depends on $u_{2}$ but $\partial v_{2} / \partial u_{2}$ does not depend on $u_{1}$.

Given these derivatives it is easy to see that the optimal investment of the firm does not depend on the government's tax rate. In the feedback Nash equilibrium the following Hamilton-Jacobi-Bellman equations hold for the two players

$$
\begin{aligned}
& \beta V_{1}(x)= \max _{\left\{u_{1}\right\}}\left\{\alpha \ln \left(\left(1-u_{1}\right) a x+w h x-u_{2}-\varphi\left(u_{2}\right)\right)\right. \\
&\left.+(1-\alpha) \ln u_{1} a x+V_{1}^{\prime}(x)\left(u_{2}-\delta x\right)\right\}, \\
& r V_{2}(x)=\max _{\left\{u_{2}\right\}}\left\{\left(1-u_{1}\right) a x-u_{2}-\varphi\left(u_{2}\right)+V_{2}^{\prime}(x)\left(u_{2}-\delta x\right)\right\} .
\end{aligned}
$$

From these equation the reaction function of each player is obtained by maximization

$$
\begin{aligned}
& u_{1}^{*}=(1 / a x)(1-\alpha)\left[1+w h x-u_{2}-\varphi\left(u_{2}\right)\right] \\
& u_{2}^{*}=\varphi^{\prime-1}\left[V_{2}^{\prime}(x)-1\right] .
\end{aligned}
$$

For the government, the tax rate and the investment are strategic substitutes, however for the firm the optimal investment only depends on the level of the state variable (capital stock).

\subsection{Strategic pigouvian taxation with stock externali- ties}

Several papers have recently been published where the authors develop a model of long-term bilateral interaction between a resource-exporting cartel 
and a coalition of governments of resource-importing countries. See Wirl (1994, 1995), Wirl and Dockner (1995), Tahvonen (1996) and Rubio and Escriche (2001). In this framework, they have studied the strategic taxation of $\mathrm{CO}_{2}$ emissions by the governments of the importing countries and thus have clarified which are the determinants of the carbon tax dynamics when the strategic behavior of the agents is taken into account. Wirl (1994) and Wirl and Dockner focus on the feedback Nash equilibrium of the game. Tahvonen studies the feedback Stackelberg equilibrium when the resourceexporting cartel acts as the leader ${ }^{11}$, and Rubio and Escriche characterize the other feedback Stackelebrg equilibrium for which the coalition of resourceimporting countries acts as the leader.

In the differential game analyzed by Rubio and Escriche, $x$ stands for cumulative emissions. The control variable of the government of the importing countries, $u_{1}$, is a tax on emissions, and the control variable of the cartel, $u_{2}$, is the producer price of the resource. The resource demand depends on the consumer price, and the dynamics of cumulative resource consumption determine simultaneously the dynamics of the $\mathrm{CO}_{2}$ concentration in the atmosphere according to the following equation ${ }^{12}$

$$
\dot{x}=a-u_{1}-u_{2,}, \square
$$

where $u_{1}+u_{2}$ is the consumer price and $a$ the independent term of the demand function.

The government of the importing countries tax emissions in order to maximize the discounted present value of the net consumers' surplus. They reimburse tax revenues as lump-sum transfers to consumers, so that the after-tax consumers' net welfare does not depend on tax revenues. The optimal time path for the tax is thus given by the solution of the following optimal control problem

$$
\begin{aligned}
& \max _{\left\{u_{1}\right\}} \int_{0}^{\infty} e^{-r t}\left(a\left(a-u_{1}-u_{2}\right)-\frac{1}{2}\left(a-u_{1}-u_{2}\right)^{2}\right. \\
& \left.-u_{2}\left(a-u_{1}-u_{2}\right)-\varepsilon x^{2}\right) d t,
\end{aligned}
$$

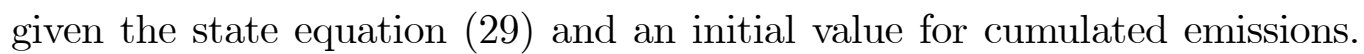
In this expression $\varepsilon x^{2}$ is the pollution damage function and the rest the net

\footnotetext{
${ }^{11}$ In Tahvonen's paper the proposed feedback Stackelberg equilibrium coincides with the feedback Nash equilibrium but the author does not explicitly recognized this because he does not calculate this last equilibrium.

${ }^{12} \mathrm{Cumulative}$ extractions can be used as a proxy of $\mathrm{CO}_{2}$ concentration if emissions are assumed irreversible.
} 
consumers' surplus. This expression can be simplified to obtain

$$
\max _{\left\{u_{1}\right\}} \int_{0}^{\infty} e^{-r t}\left(\frac{1}{2}\left(a-u_{2}\right)^{2}-\frac{1}{2} u_{1}^{2}-\varepsilon x^{2}\right) d t .
$$

On the other hand, the authors assume that extraction costs depend linearly on the rate of extraction and on the cumulative extraction. Then, the objective of the cartel of producers is to define a price strategy that maximizes the discounts present value of profits

$$
\max _{\left\{u_{2}\right\}} \int_{0}^{\infty} e^{-r t}\left(\left(u_{2}-c x\right)\left(a-u_{1}-u_{2}\right)\right) d t,
$$

given the state equation (28) and an initial value for cumulated emissions. In this last expression $c x$ is the marginal(=average) cost of extraction. This implies that extraction costs increase with cumulative extractions.

For this differential game we can check that

$$
\frac{\partial v_{1}}{\partial u_{1}}=-u_{1}, \quad \frac{\partial f}{\partial u_{1}}=-1,
$$

and

$$
\frac{\partial v_{2}}{\partial u_{2}}=a-u_{1}-2 u_{2}+c x, \frac{\partial f}{\partial u_{2}}=-1 .
$$

Then, by Corollary 1 we can conclude that there exists only one feedback Stackelberg equilibrium for the differential game for which the leader is the coalition of importing countries and the follower the cartel of exporting countries. 


\section{Conclusions}

In this paper we have investigated the scope of the applicability of the Stackelberg equilibrium concept in differential games. Our results show that for a class of differential games with state-interdependence the stationary feedback Stackelberg equilibrium coincides with the stationary feedback Nash equilibrium. For this class of differential games the strategic interdependence among the agents occurs only through the dynamics of the sate variable. Then, since the asymmetry in the roles of the players in a continuous-time feedback Stackelberg equilibrium is only incremental, the feedback Stackelberg equilibrium is identical to the feedback Nash equilibrium. Nevertheless, this result is more general and applies for a broader class of differential games. A sufficient condition for getting this coincidence is that the reaction functions of the players be orthogonal with respect to the control variables. A review of different economic applications of differential games shows that the feedback Stackelberg equilibrium is identical to the feedback Nash equilibrium for a good number of interesting economic applications what means that this result is pretty general. At least, it is going to appear in all the economic models in continuous time with stock externalities that cover issues as the global warming problem, the exploitation of common property resources, both renewable and non-renewable, the dynamic provision of public goods and the competitive arms race.

This result restricts the applicability of the Stackelberg equilibrium concept in differential games. In order to avoid this restriction the calculation of non-degenerate feedback Stackelberg equilibria has been proposed, see Dockner at el. (2000, 5.3). However, this procedure also presents some problems. Mainly, using this approach one solves a game with feedback strategies for the follower but with open-loop strategies for the leader. Another alternative is to change the framework and use a discrete-time approach for the analysis of leadership in a dynamic setting, see Başar and Olsder (1999, Chap. 7). 


\section{References}

[1] Başar, T., Haurie, A. and Ricci, G. (1985), "On the dominance of capitalism leadership in a 'feedback-Stackelberg' solution of a differential game model of capitalism", Journal of Economics Dynamics and Control 9:101-25.

[2] Başar, T. and Olsder, G.J. (1999), Dynamic Noncooperative Game Theory, 2nd ed., Philadelphia: SIAM.

[3] Benhabib, J. and Radner, R. (1992), "The joint exploitation of a productive asset: A game-theoretic approach", Economic Theory 2:155-90.

[4] Brito, D.L. (1972), "A dynamic model of an arms race", International Economic Review 13:359-75.

[5] Brito, D.L. and Intriligator, M.D. (1987), "Stock externalities, pigovian taxation and dynamic stability", Journal of Public Economics 33:59-72.

[6] Deger, S. and Sen, S. (1984), "Optimal control and differential game models of military expenditure in less developed countries", Journal of Economic Dynamics \& Control 7:153-69.

[7] Dockner, E.J. (1992), "A dynamic theory of conjectural variations", Journal of Industrial Economics 49:377-95.

[8] Dockner, E.J. and Long, N.V. (1993), "International pollution control: Cooperative versus noncooperative strategies", Journal of Environmental Economics and Management 24:13-29.

[9] Dockner, E.J. and Sorger, G. (1996), "Existence and properties of equilibria for a dynamic game on productive assets", Journal of Economic Theory 71:209-27.

[10] Dockner, E.J., Feichtinger, G. and Mehlmann, A. (1989), "Noncooperative solutions for a differential game model of fishery", Journal of Economic Dynamics \& Control 13:1-20.

[11] Dockner, E.J., Jorgensen, S., Long, N.V. and Sorger, G. (2000), Differential Games in Economics and Management Science, Cambridge, U.K.: Cambridge University Press.

[12] Driskill, R.A. and McCafferty, S. (1989), "Dynamic duopoly with adjustment costs: A differential game approach", Journal of Economic Theory 49:324-38. 
[13] Fershtman, C. and Muller, E. (1984), "Capital accumulation games of infinite duration", Journal of Economic Theory 33:322-39.

[14] Fershtman, C. and Kamien, M.I. (1987), "Dynamic duopolistic competition with sticky prices", Econometrica 55:1987.

[15] Fershtman, C. and Nitzan, S. (1991), "Dynamic voluntary provision of public goods", European Economic Review 35:1057-67.

[16] Gisser, M. and Sánchez. D.A. (1980), "Competition versus optimal control in groundwater pumping", Water Resources Research16:638-42.

[17] Gradus, R. (1991), "Optimal dynamic profit taxation: The derivation of feedback Stackelberg equilibria", Metroeconomica 42:157-77.

[18] Itaya, J. and Shimomura, K. (2001), "A dynamic conjectural variations model in the private provision of public goods: A differential game approach", Journal of Public Economics 81:153-72.

[19] Kamien, M.I. and Schwartz, N.L. (1991), Dynamic Optimization. The Calculus of Variations and Optimal Control in Economics and Management, 2nd ed., Amsterdam:North-Holland.

[20] Levhari, D. and Mirman, L.J. (1980), "The great fish war: An example using a dynamic Cournot-Nash solution", Bell Journal of Economics and Management Science 11:322-34.

[21] List, J.A. and Mason, C.F. (2001), "Optimal institutional arrangements for transboundary pollutants in a second-best world: Evidence from a differential game with asymmetric players", Journal of Environmental Economics and Management, forthcoming.

[22] Long, N.V. (1992), "Pollution control: A differential game approach", Annals of Operations Research 37:283-96.

[23] Maskin, E. and Tirole, J. (1987), "A theory of dynamic oligopoly, III. Cournot competition", European Economic Review 31:947-68.

[24] Negri, D.H. (1989), "The common property aquifer as a differential game", Water Resources Research 25:9-15.

[25] Piga, C.A.G. (2000), "Competition in a duopoly with sticky price and advertising", International Journal of Industrial Organization 18:595614. 
[26] Ploeg, F. van der and de Zeeuw, A.J. (1990), "Perfect equilibrium in a model of competitive arms accumulation", International Economic Review 31:131-46.

[27] Ploeg, F.van der and de Zeeuw, A. J. (1992), "International aspects of pollution control", Environmental and Resource Economics 2:117-39.

[28] Reynolds, S.S. (1987), "Capacity investment, preemption and commitment in an infinite horizon model", International Economic Review 28:69-88.

[29] Reynolds, S.S. (1991), "Dynamic oligopoly with capacity adjustment costs", Journal of Economic Dynamics \& Control 15:491-514.

[30] Rubio, S.J. and Casino, B. (2001), "Competitive versus efficient extraction of a common property resource: The groundwater case", Journal of Economic Dynamics \& Control 25:1117-37.

[31] Rubio, S.J. and Escriche, L. (2001), "Strategic pigouvian taxation, stock externalities and polluting non-renewable resources", Journal of Public Economics 79:297-313.

[32] Simaan, M. and Cruz, J.B. Jr. (1975), "Formulation of Richardson's model of arms race from a differential game viewpoint", Review of Economic Studies 42:67-77.

[33] Tahvonen, O. (1996), "Trade with polluting nonrenewable resources", Journal of Environmental Economics and Management 30:1-17.

[34] Tsutsui, S. (1996), "Capacity constraints and voluntary output cutback in dynamic Cournot competition", Journal of Economic Dynamics $\&$ Control 20:1683-708.

[35] Tsutsui, S. and Mino, K. (1990), "Nonlinear strategies in dynamic duopolistic competition with sticky prices", Journal of Economic Theory 52:136-61.

[36] Wirl, F. (1994), "Pigouvian taxation of energy for flow and stock externalities and strategic, noncompetitive energy pricing", Journal of Environmental Economics and Management 26:1-18.

[37] Wirl, F. (1995), "The exploitation of fossil fuels under the threat of global warming and carbon taxes: A dynamic game approach", Environmental and Resource Economics 5:333-52. 
[38] Wirl, F. and Dockner, E.J. (1995), "Leviathan governments and carbon taxes: Costs and potential benefits", European Economic Review 39:1215-36.

[39] Zagonari, F. (1998), "International pollution problems: Unilateral initiatives by environmental groups in one country", Journal of Environmental Economics and Management 36:46-69. 